\title{
DEL CONTROL TERRITORIAL AL INDIVIDUO: UN CAMINO HACIA EL ACTUARIALISMO*
}

\author{
Julio Alberto Novoa Ruíz ${ }^{1}$
}

\section{RESUMEN}

El control actuarial es de imperiosa utilización actual, toda vez que la concentración de acciones criminales que realizan las diferentes organizaciones al margen de la ley (guerrillas, bandas criminales y narcotraficantes), que también han desplegado su presencia a los centros urbanos, avizora la necesidad de diseñar estrategias de control actuarial en la prevención de seguridad por parte de la fuerza pública, con herramientas tecnológicas e informáticas, a fin de ejercer un adecuado control sobre la población lugareña.

\section{PALABRAS CLAVES}

Actuarial, fuerza, pública, informática, tecnología.

\begin{abstract}
The actuarial control is a much needed form of control nowadays because of the increase of criminal actions executed by outlaw organizations (guerrillas, criminal groups, and drug dealers), which, also are spreading through the urban areas, this prompts the necessity to design new strategies of actuarial control in order to guarantee safety by the security forces with technologic and informatics tools in order to exercise an adequate control over the population from the villages.
\end{abstract}

\section{KEYWORDS}

Actuarial, Security Forces, Informatics, Technology

Depositado en febrero 17 de 2014, aprobado en mayo 23 de 2014.

* Este documento es un extracto de la investigación La Cuestión Integral del siglo XXI, en la cual el autor plantea cómo el conflicto colombiano ha hecho un movimiento de control territorial desde lo rural hacia lo urbano, y cómo se presenta el Actuarialismo en el control del individuo.

1 Magister en Negocios y Relaciones Internacionales. Especializado en Seguridad y Defensa Nacional, Sociología de las Relaciones Internacionales, Derechos Humanos y Resolución de conflictos. Administrador de Empresas y Docente Especial. 


\section{INTRODUCCIÓN}

En la actualidad, las amenazas que afectan el funcionamiento burocrático del Estado, así como a la sociedad que lo conforma (Weber, 1994) se mantienen, aunque su forma de actuar cambia en la medida de sus necesidades, bien sea para preservar su infraestructura física como organización al margen de la ley o para garantizar su gestión y obtener réditos políticos o económicos. En este mismo sentido, el aumento de su accionar criminal en su empeño por obtener sus logros ha influenciado a las mencionadas organizaciones para que su presencia sea más fuerte en los centros urbanos, sin importar su tamaño, puesto que en ellos se concentra numerosa población que actúa como receptora de sus productos, sin dejar de lado la periferia rural que es su garantía para mantener su presencia como infraestructura física organizada y a la vez, mantener el flujo ideológico en el caso de las guerrillas y los narcóticos procesados para el caso de las bandas criminales y narcotraficantes.

En consecuencia, se observan numerosas acciones criminales por parte de las diferentes organizaciones al margen de la ley, en el centro o en las cabeceras municipales. Estas acciones tienen su origen en el área rural y por lo tanto, es necesario desarrollar estrategias de control actuarial para modificar el ambiente mismo en donde existen las potenciales organizaciones, y desde donde se generan el delito o acción criminal, así como adecuar el control necesario y específico para adecuarlo a la población de los centros urbanos donde se presenten.

El control actuarial afecta necesariamente las tres dimensiones que comprende el escenario de donde proviene la acción criminal. En tal sentido, una prevención de seguridad primaria se orienta hacia las potenciales comunidades desde donde se pueda desarrollar y proyectar acciones criminales, y debe ser afectada en su medio para evitar el comienzo de tales acciones. Una prevención de seguridad secundaria está dirigida a identificar las organizaciones al margen de la ley, y una prevención de seguridad terciaria está orientada hacia los líderes de las organizaciones delincuenciales. Mientras que la prevención primaria debe afectar el área geográfica, la prevención secundaria y la terciaria constituyen el esfuerzo que se orienta hacia el individuo que actúa como generador racional de la acción criminal o delito.

Con el objeto de que los controles actuariales desarrollados por parte de la fuerza pública tengan efectividad sobre el individuo, deben acompañarse de herramientas tecnológicas e informáticas mediante la concentración de información, a fin de conocer su comportamiento, sus proyecciones, actividad económica, etc. El empleo de estas herramientas de control hacen parte del concepto Actuarial, objeto de estudio de este trabajo investigativo.

\section{DE LO RURAL A LO URBANO}

Por tradición, la dinámica operacional de los diferentes ejércitos del Mundo, ha sido la de retener territorio, reconocer sus territorios y por lo tanto, sus fronteras (Patiño, 2013: 9. 29), para obtener acceso a los mares, a los ríos y a las riquezas minerales y se constituye entonces, en fuente de conquistas para favorecer los intereses de los invasores. Hasta el presente, las experiencias de los diferentes imperios, incluida la promoción de mecanismos legales diferentes a la guerra, como el del referéndum con el cual Crimea se independizó de Ucrania y favoreció los intereses de la Federación Rusa, no ha sido la excepción ahora en el siglo XXI (2014, 23 de marzo).

Pensar en la globalización como la única forma actual de poder ocupar e influenciar las mentes y los intereses económicos de las decisiones de los gobernantes para cambiar el comportamiento de sus ciudadanos, parece compartirse con otras modalidades como el populismo de izquierda (Torres y Peruzzotti, 2008: 27), y el autoritarismo que se presenta en países como Venezuela, Nicaragua, Ecuador, Bolivia y Argentina, para lograr el control de una América Latina con la modalidad ideológica de la época de la guerra fría. Así entonces, se evidencia con preocupación que en el Mundo, todavía se presentan casos de pretensión del control territorial con el uso de la fuerza, de las ideologías y de la independencia promovida por sentimientos 
étnicos, con el objetivo de proyectar desde estas nuevas conquistas lo que se considera para los diferentes gobernantes, la forma de ejercer y prolongar el control sobre el territorio y sobre los pueblos que lo habitan.

Queda entonces, que los elementos constitutivos de la existencia del Estado, esto es, el territorio, la población y el poder, tienen la necesidad de interactuar de manera interdependiente, jugando un papel determinante para obtener el bienestar de su población (MinDefensa, 2006). Cobra así especial atención, definir la forma de garantizar el logro del fin del Estado, el bien común público, que los gobernantes propenden al diseñar estrategias para lograr el interés nacional, pues mientras existan los Estados soberanos, siempre estarán expuestos a la posibilidad de la confrontación (Waltz, 2007).

En América Latina, todavía existen conflictos internos como producto de la guerra fría, en especial en Colombia, lo cual obligó a Estados Unidos a diseñar la estrategia nacional identificada como "Doctrina de Seguridad Nacional", en la cual se incluyó como principal enemigo el comunismo internacional con epicentro en la Unión Soviética y con representación regional en Cuba. En aquel entonces, los Estados latinoamericanos debían enfrentar al enemigo interno, materializado en supuestos agentes locales del comunismo. Además de las guerrillas, el enemigo interno podía ser cualquier persona, grupo o institución nacional que tuviera ideas opuestas a las de los gobiernos militares (Leal, 2003: 75).

Es urgente por lo tanto, diseñar una estrategia de seguridad que defina la transición del control del territorio, al control directo de la población, en el entendido de que aún persiste un conflicto interno, en donde las partes comprometidas son las guerrillas terroristas (FARC y ELN), bandas criminales, narcotraficantes, contrabandistas que prevalecen en el enfrentamiento contra el Estado y la población. La necesidad de controlar todas estas amenazas exige hacer un esfuerzo contra los individuos que las integran, puesto que el escenario territorial que incluye la porción marítima, fluvial y aérea, en donde se desarrolla toda actuación criminal, es ocasionada por las personas como actos racionales, mas no como causas sociales que conlleven el desarrollo de toda acción criminal, a fin de justificar su existencia y mantener su supervivencia ( $\mathrm{O}^{\prime}$ Malley, 2004).

Además, es fundamental que la estrategia de seguridad deba considerar un concepto holístico, en donde el sistema de seguridad de prevención -objeto de estudio de este artículo-, se entrelace con el sistema de justicia y el penitenciario, para lo cual se ha denominado como la "Cuestión Integral"2 (Novoa, 2013: 168). Se considera entonces, que el estado del arte nos lleve a entender el concepto de estudio sobre el control del individuo, partiendo de manera centrífuga de fuera hacia adentro, desde el control territorial para llegar hasta el propio individuo criminal.

\section{PREVENCIÓN SITUACIONAL}

La condición geográfica de los países en donde persiste el conflicto, se ha caracterizado por condiciones difíciles, con alturas, depresiones, selvas y numerosos ríos caudalosos, lo cual hace que su alteración para facilitar su control sea imposible, debido a la afectación de los ecosistemas. Pero la alteración del medio ambiente desde donde se pueda originar la acción criminal, es lo que se denomina según De Giorgi (2004), la prevención situacional y consiste en desarrollar acciones tendientes a modificar las condiciones del medio ambiente que propician, para el caso colombiano, acciones de criminalidad, la cadena de infraestructura del narcotráfico (cultivos, químicos, laboratorios, rutas de distribución, distribuidores, comercializadores, vendedores, etc.), lo mismo que la cadena de las guerrillas (campamentos, rutas de desplazamiento, acceso a poblaciones, identificación de cabecillas, identificación de sus milicias, objetivos militares de las guerri-

2 La cuestión integral es un concepto actual que se quiere incluir en el tema actuarial, en donde se pretende entrelazar el sistema de seguridad de prevención con el sistema de justicia y el penitenciario. 
llas, etc.), sobre la infraestructura energética, vial, poblaciones, centros económicos, etc. Lo particular de la prevención situacional es que se abandonan las preocupaciones por el tema criminal (las guerrillas, los narcotraficantes, los terroristas y los contrabandistas), y se centra sólo en buscar los medios para poder controlar la acción criminal.

El concepto de la prevención situacional no puede enmarcarse como una teoría criminológica, puesto que está orientada a dirigir el esfuerzo de la fuerza pública a las condiciones ambientales para prevenir la acción de las diferentes clases de delincuentes. Este aspecto se considera como una prevención primaria que afecta el inicio y desarrollo de acciones desde donde se pueda originar delito o acción criminal que puedan atentar contra la comunidad y al mismo tiempo, el esfuerzo se encamina a identificar los grupos poblacionales como son las bandas criminales, las organizaciones terroristas, las redes de narcotráfico y otros, a partir de los cuales se originan las acciones criminales. Esto es lo que se llama la prevención secundaria, debido a que las diferentes organizaciones criminales poseen una infraestructura conformada por individuos que como organización delincuencial desarrollan diferentes acciones tendientes a fortalecer su propia infraestructura y la de su accionar. De tal manera que una vez se pueda conocer su infraestructura, su accionar y sus propósitos, es decisivo identificar los potenciales líderes para neutralizar su actividad de forma directa, labor exigente para la fuerza pública, pero a la cual se debe el mayor esfuerzo para desarticular las acciones criminales de hoy y del mañana, todo lo cual se denomina prevención terciaria. Ahora bien, el diseño de los mecanismos y las herramientas para poder generar el control sobre el territorio, los grupos poblacionales y sus líderes que aún condenados y encarcelados continúan siendo generadores de riesgo (Rutherford, 2000: 58), se podrá entender entonces el concepto Actuarial ${ }^{3}$, del cual trata el siguiente apartado.

La prevención situacional es propia de la doctrina criminal y se puede considerar para la acción de la fuerza pública, puesto que las acciones generadas por las diferentes organizaciones al margen de la ley, son acciones tendientes a concretar el delito, sin importar si es político o no. En realidad, lo que interesa a la sociedad es el daño que le produzca, si se convierte en reiterativo y si la conducta del criminal se convierte en reincidencia, lo cual obliga a que el Estado mediante su fuerza pública, diseñe estrategias encaminadas a neutralizar las acciones que afecten directamente la Seguridad y la Defensa Nacional, lo mismo que la referida tranquilidad ciudadana.

\section{ACTUARIALISMO}

Para desarrollar esta sección del trabajo, es conveniente incluir conceptos sobre justicia criminal, porque la respuesta al accionar de los criminales en los diferentes escenarios, como la agresión de la delincuencia común y organizada contra la sociedad, como la de la guerra para el caso de Colombia, la de las guerrillas contra la misma comunidad y el Estado, ha evolucionado debido a la misma sociedad global en donde el riesgo y la gestión del riesgo es el producto propio de la sociedad industrializada (Beck, 1998-2002), e influye en el desarrollo de otros grandes peligros como los conflictos internos o la misma guerra, los cuales están siendo dinamizados por individuos que hacen parte de los grupos poblacionales que originan acciones criminales contra una sociedad.

Según Rivera (2009), con la nueva penología ${ }^{4}$ se reorienta el interés del sistema penal, trasladando el individuo a un concepto de manejo de grupos poblacionales, en donde el objetivo de la justicia se desarrolla mediante controles e identificaciones, como portadores del riesgo en sus diferentes niveles de amenaza (actuarial). Con

\footnotetext{
3 Según el Diccionario RAE, actuarial es un adjetivo que significa "relativo al actuario de seguros o a sus funciones", es decir, relacionado con cálculos matemáticos y conocimientos estadísticos, jurídicos y financieros concernientes a los seguros y a su régimen, propio de las entidades aseguradoras.

4 La Nueva Penología busca regular los niveles de desviación sin intervenir ni responder a individuos desviados o a las malformaciones sociales (Feeley y Simon: 36).
} 
el desarrollo de este nuevo concepto, el manejo del riesgo en el campo penitenciario, se hace ejerciendo un control por medio de técnicas de vigilancia por medio de estadísticas de clasificación y agrupación, con base en su potencial desestabilizador (Rivera, 2009: 340).

Se tiene entonces, un modelo que comienza a buscar efectos sobre la población criminal, empleando tecnologías y nuevos procedimientos que involucra a la sociedad civil, permitiéndole ser los ojos para coadyuvar en el mismo control a las diferentes autoridades encargadas de aplicar los mecanismos de control y vigilancia (Novoa, 2012: 77).

Así como lo tiene establecido Rivera, la justicia actuarial no piensa en términos de culpabilidad sino de riesgo, pues los conflictos son la consecuencia de la modernidad e industrialización, y se manifiestan por sectores poblacionales o de clases, en donde no queda margen para que se siga individualizando las responsabilidades o culpabilidades en los individuos. Esto se debe a que el mismo sistema de política criminal, penitenciaria y de prevención de la seguridad, no es suficiente para controlar el enorme escenario de progreso del riesgo. Por consiguiente, se considera que el esfuerzo de las autoridades está orientado a identificar y clasificar los riesgos que potencialmente generen delito, y a determinar el grupo poblacional a donde pertenecen, para observar así, la efectividad del modelo actuarial (Rivera, 2009: 341).

Es imperioso conocer sobre el managerialismo ${ }^{5}$ como una expresión clara de la sociedad del control del riesgo que no se preocupa por mejorar, sino por gestionar, en donde la criminología se ha transformado en un tema del discurso, generalizando el análisis y la implementación de políticas públicas, lo mismo que la manejar situaciones más o menos problemáticas.

En cuanto a la justicia actuarial, Bergalli sostiene que al igual que todos los sistemas de control del delito, requiere credibilidad y legitimidad ante la ciudadanía para permitir un funciona- miento estable y un control efectivo sobre la criminalidad (Leal, 2004). Es de suponer que sin los elementos expuestos anteriormente, no se produciría un efecto de control y de transparencia, de manera que la misma estrategia actuarial es la que pretende legitimarse ante la población, difundiendo un discurso lleno de contenido simbólico de peligrosidad social, de grupos y zonas de riesgo, etc. (Bergalli, 2001).

Es preciso entender la necesidad de una perfecta alineación entre las políticas públicas de seguridad, criminal y penitenciaria, y una sinergia ágil que corresponda al actuarialismo (control del riesgo), en cada eslabón que compone el gran sistema de la Cuestión Integral. Visto de otra manera, la actuación de la fuerza pública (para el caso de Colombia por la Policía Nacional y las Fuerzas Militares), el diseño de la política criminal para atenuar el crimen y la delincuencia, lo mismo que la política penitenciaria en la cual se debe fortalecer la administración y el comportamiento del servicio de vigilancia interna, como indiscutible comportamiento de la población reclusa, al cual van dirigidos los procesos y el concepto actuarial de control dentro de los establecimientos carcelarios y penitenciarios.

La Cuestión lntegral es el llamado continuo de la sociedad al Estado para garantizar la protección de los intereses del mismo y de los residentes del territorio bajo su jurisdicción, los cuales necesitan de una organización adecuada para que los ciudadanos y el mismo Estado, se identifiquen y hagan una causa común para enfrentar las diferentes amenazas que permanecen asediando el desenvolvimiento de la Nación (Novoa, 2012).

Lo anterior ha servido de enlace al salto que se necesita para exponer cómo la influencia del Actuarialismo sobe el sistema penitenciario y el de la seguridad, donde convergen las acciones que la fuerza pública desarrolla tanto en el área rural como en el área urbana de Colombia, por ser el escenario propio para generar acciones actuariales a fin de contrarrestar las amenazas

5 El managerialismo es un término que proviene de manager e indica administrar el riesgo. 
que se han desplazado desde la periferia hacia el centro de las cabeceras municipales, puesto que allí es donde se concentra la mayoría de acciones criminales de las guerrillas, los narcotraficantes, las bandas criminales, y la delincuencia común y organizada (Tablas 1, 2 y 3 ).

Tabla No. 1. Delitos delincuencia común, colectiva, secuestros 2008-2014*

\begin{tabular}{|l|l|l|l|l|l|l|l|}
\hline DELITO & $\mathbf{2 0 0 8}$ & $\mathbf{2 0 0 9}$ & $\mathbf{2 0 1 0}$ & $\mathbf{2 0 1 1}$ & $\mathbf{2 0 1 2}$ & $\mathbf{2 0 1 3}$ & $\mathbf{2 0 1 4}$ \\
\hline $\begin{array}{l}\text { Homicidio } \\
\text { común }\end{array}$ & 16.140 & 15.817 & 15.459 & 16.127 & 16.440 & 15.234 & 5.386 \\
\hline $\begin{array}{l}\text { Homicidio } \\
\text { colectivo }\end{array}$ & 169 & 147 & 183 & 171 & 157 & 104 & 25 \\
\hline Secuestros & 437 & 213 & 282 & 305 & 305 & 299 & $\mathbf{1 1 2}$ \\
\hline
\end{tabular}

Fuente: Ministerio Defensa Nacional. Datos a mayo de 2014.

\section{ACCIONES ACTUARIALES}

El ritmo operacional que han impuesto las Fuerzas Militares y la Policía Nacional en las áreas urbanas ha sido muy contundente hasta la fecha, pero se cree que la modalidad criminal de las organizaciones narco terroristas se ha trasladado en gran parte, del área rural a la urbana, bajo la modalidad de desplazamientos justificados por el conflicto, y con la búsqueda de mejores oportunidades como supervivencia y desarrollo para los núcleos familiares, pero al mismo tiempo, se evidencia el fortalecimiento de la cadena de la empresa del narcotráfico, con la distribución, comercialización y consumo, dejando el cultivo y el procesamiento de la pasta de coca al área rural, puesto que se re- quiere controles tecnológicos para rastrear los insumos (químicos, gasolina, cemento, etc.), necesarios para llevar a cabo el procesamiento. Sin embargo, el producto debe salir a las áreas urbanas bien sea para su comercialización o distribución a los diferentes países del Mundo.

El seguimiento a los insumos debe hacerse necesariamente con medios tecnológicos como son los códigos de barras y la radiofrecuencia para conocer la trazabilidad. No se puede ejercer control sobre estos elementos en la forma como se ha hecho hasta ahora, es decir, de manera empírica, en donde no se les exige a las fábricas productoras que permitan el control. Ahora bien, la fuerza pública requiere herramientas tecnológicas para leer los diferentes insumos.

Tabla No. 2. Delitos hurto común (automotores, motocicletas).

Hurto común (residencias, comercio y personas). 2008-2014*

\begin{tabular}{|l|l|l|l|l|l|l|l|}
\hline DELITO & $\mathbf{2 0 0 8}$ & $\mathbf{2 0 0 9}$ & $\mathbf{2 0 1 0}$ & $\mathbf{2 0 1 1}$ & $\mathbf{2 0 1 2}$ & $\mathbf{2 0 1 3}$ & $\mathbf{2 0 1 4}$ \\
\hline $\begin{array}{l}\text { Hurto } \\
\text { Vehículos }\end{array}$ & 19.637 & 21.550 & 21.442 & 30.706 & 31.860 & 31.491 & 11.882 \\
\hline $\begin{array}{l}\text { Hurto } \\
\text { Común }\end{array}$ & 104.418 & 94.288 & 92.273 & 101.308 & 126.516 & 131.091 & 54.466 \\
\hline
\end{tabular}

Fuente: Ministerio Defensa Nacional. Datos a mayo de 2014.

El objetivo de la investigación es orientar el esfuerzo de la fuerza pública hacia el control de las personas, por ser ellas las que ocasionan el delito, como ya se dijo unas líneas atrás. Para el caso de las guerrillas, el delito es un acto racional y no obedece a causas sociales ni ideo- lógicas; entonces, el control actuarial que se debe hacer, se soporta con una base de datos concentrada en una central de inteligencia y que como ya se dijo, debe alimentarse con una aplicación App que debe ser creada por tecnólogos informáticos de la propia Fuerza, por interme- 
dio de medios de telefonía celular móvil y que debe contener datos biográficos, fotografía, dirección de residencia, coordenadas de residen- cia, vehículo, conductor, actividad económica, estudios, etc., para censar la población y conocer su actividad.

Tabla No. 3. Actos terroristas contra la infraestructura (oleoductos, torres energía, vías, puentes). 2008-2014*

\begin{tabular}{|l|l|l|l|l|l|l|l|}
\hline $\begin{array}{l}\text { ACCIONES } \\
\text { TERRORISTAS }\end{array}$ & $\mathbf{2 0 0 8}$ & $\mathbf{2 0 0 9}$ & $\mathbf{2 0 1 0}$ & $\mathbf{2 0 1 1}$ & $\mathbf{2 0 1 2}$ & $\mathbf{2 0 1 3}$ & $\mathbf{2 0 1 4}$ \\
\hline Oleoductos & 32 & 32 & 31 & 84 & 151 & 259 & 57 \\
\hline Torres energía & 138 & 77 & 39 & 73 & 47 & 108 & 5 \\
\hline Puentes & 3 & 1 & 1 & 1 & 6 & 5 & 2 \\
\hline Vías & 87 & 46 & 45 & 38 & 75 & 36 & 23 \\
\hline
\end{tabular}

Fuente: Ministerio Defensa Nacional. Datos a mayo de 2014.

La responsabilidad del manejo de esta información, es exclusiva de los comandantes que con registros informáticos establecen el acceso al sistema para alimentarlo y no se puede permitir que la fuerza pública continúe su labor sin ayuda de herramientas tecnológicas que faciliten el control actuarial sobre los individuos de generan delitos. Puesto que bajo ningún punto de vista se puede violar la privacidad de las personas, es necesario establecer una normatividad para no vulnerar los derechos de las personas.

El incremento de la actividad criminal por parte de las guerrillas, bandas criminales, narcotraficantes, delincuencia organizada y común se ha orientado hacia las cabeceras municipales y áreas suburbanas de las mismas, lo cual implica desarrollar una estrategia actuarial donde participen la Policía Nacional y las Fuerzas Militares para enfrentar estas amenazas que afectan la Seguridad y la Defensa Nacional, sin dejar espacios territoriales que distraigan a la fuerza pública, y desarrollen su accionar delictivo por falta de estrategias de control actuarial.

En ningún momento se menciona el traslado completo del esfuerzo principal que hacen las Fuerzas Militares sobre el control territorial, en especial en las áreas rurales, puesto que allí se encuentra el inmenso potencial económico que provee de productos primarios y fortalece la economía del País. Allí también se evidencian las acciones por de los diferentes Grupos Delincuenciales al Margen de la Ley
(Tabla 3), y que traen consigo el deterioro de la economía y por supuesto, afecta la seguridad. Lo anterior fortalece el concepto actuarial de ejercer un control actuarial y lograr identificar comunidades potenciales que generan delitos, organizaciones delincuenciales y sus líderes, con el fin de adelantar acciones militares para neutralizarlas.

Si bien, las estadísticas (Tablas 5 y 6), muestran la acción contundente de la fuerza pública que si bien ha desarrollado un trabajo impecable para combatir las diferentes amenazas, también es cierto que es necesario revisar la orientación para ejercer un control actuarial mayor en las áreas urbanas y suburbanas, puesto que allí se concentra la mayor cantidad de delitos y acciones por parte de las diferentes amenazas que deterioran la seguridad y la tranquilidad ciudadanas.

El fin principal del actuarialismo es precisamente, afectar el medio ambiente donde se crean y delinquen las organizaciones delincuenciales, para evitar que se produzca la acción criminal o delito, pero al mismo tiempo, se considera identificar y clasificarlos para orientar los esfuerzos de la fuerza pública, con el fin de afectar e impedir que se produzca una acción criminal y al mismo tiempo, se soporta en un elemento muy importante, la tecnología e informática, herramientas que facilitan en tiempo real, conocer el comportamiento de los individuos que integran los grupos criminales y neutralizarlo. 
Tabla No. 4. Acciones de grupos armados al margen de la ley (ataque aeronaves, instalaciones, hostigamiento, emboscadas, contacto armado, retenes ilegales). 2008-2014*

\begin{tabular}{|l|l|l|l|l|l|l|l|}
\hline ACCIONES & $\mathbf{2 0 0 8}$ & $\mathbf{2 0 0 9}$ & $\mathbf{2 0 1 0}$ & $\mathbf{2 0 1 1}$ & $\mathbf{2 0 1 2}$ & $\mathbf{2 0 1 3}$ & $\mathbf{2 0 1 4}$ \\
\hline Acciones & 52 & 161 & 131 & 152 & 167 & 160 & 74 \\
\hline
\end{tabular}

Fuente: Ministerio Defensa Nacional. Datos a mayo de 2014.

El diseño de software y las aplicaciones Apps son algunos de los mecanismos que se emplean para manejar la información, puesto que es evidente que la fuerza pública carece de estos mecanismos para identificar y ubicar los potenciales generadores de delito. Ahora bien, esto no puede implicar la violación de la privacidad de las personas, porque la fuerza se expone a cuestionamientos de la misma sociedad que no debe permitirse.

El actuarialismo es sinónimo de control y tecnología. Como no se puede pretender que la fuerza pública sea más efectiva sin tener estas herramientas que pueden diseñarse con los más altos estándares de calidad y por supuesto, por los mismos integrantes de las fuerzas, requiere a la vez un control y centralización por parte del alto Mando, para diseñar directrices, seguridad informática y manejo de la información.

En los últimos cuatro años, los resultados operacionales de la fuerza pública han mantenido una constante que le da una impronta de efec- tividad ante las amenazas que se reproducen a diario por parte de las organizaciones al margen de la ley, pero al mismo tiempo, es importante poder contrarrestarles su acción con la implementación de controles actuariales dirigidos en especial hacia sus integrantes, para evitar y neutralizar su actividad y garantizar tranquilidad y seguridad a la sociedad, puesto que la misma confrontación armada no es suficiente porque la criminalidad ha desarrollado diferentes formas de actuar, lo cual exige diseñar estrategias actuariales de control, empleando tecnología e informática para afectar el ambiente propio en donde se desenvuelve la criminalidad.

Las acciones actuariales de control que afectan el hábitat de las organizaciones al margen de la ley, se hacen efectivas con el desarrollo de mecanismos tecnológicos que ejerzan control sobre la misma población. Es indispensable conocer con anticipación el potencial desarrollo criminal de estas organizaciones, porque la concepción reactiva dificulta que la fuerza pública

Tabla No. 5. Resultados operacionales Fuerza Pública (FARC, ELN EPL, ERG, JBC). 2008-2014*

\begin{tabular}{|l|l|l|l|l|l|l|l|}
\hline RESULTADO & $\mathbf{2 0 0 8}$ & $\mathbf{2 0 0 9}$ & $\mathbf{2 0 1 0}$ & $\mathbf{2 0 1 1}$ & $\mathbf{2 0 1 2}$ & $\mathbf{2 0 1 3}$ & $\mathbf{2 0 1 4}$ \\
\hline Desmovilizados & 3.461 & 2.538 & 2.446 & 1.527 & 1.140 & 1.350 & 553 \\
\hline Capturas & 2.483 & 2.252 & 1.808 & 2.059 & 3.151 & 2.611 & 1.271 \\
\hline Muertos combate & 1.184 & 584 & 493 & 373 & 439 & 340 & 136 \\
\hline
\end{tabular}

Fuente: Ministerio Defensa Nacional. Datos a mayo de 2014.

Tabla No. 6. Resultados operacionales Fuerza Pública (BACRIM)*

\begin{tabular}{|l|l|l|l|l|l|l|}
\hline RESULTADO & $\mathbf{2 0 0 9}$ & $\mathbf{2 0 1 0}$ & $\mathbf{2 0 1 1}$ & $\mathbf{2 0 1 2}$ & $\mathbf{2 0 1 3}$ & $\mathbf{2 0 1 4}$ \\
\hline Capturas & 2.935 & 3.366 & 3.817 & 4.493 & 3.894 & 1.187 \\
\hline Muertos combate & 51 & 52 & 43 & 43 & 33 & 13 \\
\hline
\end{tabular}

Fuente: Ministerio Defensa Nacional. Datos a mayo de 2014. 
actúe con claridad. Es el caso de la prevención situacional, orientada hacia las potenciales comunidades gestoras de criminalidad, con mayor énfasis en las organizaciones al margen de la ley identificadas, así como a sus líderes, lo cual garantiza no sólo la efectividad de la fuerza pública, sino la razón sine qua non de garantizar seguridad y tranquilidad ciudadanas.

En el presente trabajo de investigación, sólo se consideraron los principales delitos contra la población y la infraestructura energética y vial, pero no se consideraron los delitos contra los recursos naturales y el medio ambiente, contra la protección a la familia ni tampoco las acciones por parte de la fuerza pública, puesto que el objeto del estudio está orientado a la necesidad de evidenciar que las acciones por parte de las diferentes amenazas contra el Estado, la infraestructura y la población, están en una relación mayor en las áreas urbanas que en las rurales.

Lo anterior implica desarrollar estrategias tendientes a conseguir el control actuarial con un mayor énfasis en las áreas urbanas, pues allí es donde se han concentrado las actividades criminales debido al desplazamiento natural y por razones del conflicto, la movilización de la población de la periferia hacia los diferentes centros urbanos.

\section{CONCLUSIONES}

Es evidente que la acción de la mayoría de las diversas amenazas se ha incrementado en las áreas urbanas y suburbanas, dejando una logística primaria pero importante en el área rural, en especial para las guerrillas y los narcotraficantes, y que el resultado de homicidios y secuestros propiciado por aquellos se ha desarrollado más en el área urbana, sin dejar de lado la otra parte contundente de las Fuerzas Militares en el área rural (Tablas 5 y 6), que sin restarle importancia, es menor en las áreas rurales pero en el entendido de que el control territorial en ellas es muy dispendioso por las distancias y su geografía, lo cual exige un compromiso mayor con respecto del número de la fuerza pública.

Ahora bien, la necesidad de implementar medios tecnológicos de control actuarial para ejercer el control sobre los individuos que originan la acción delincuencial y terrorista de las diferentes organizaciones al margen de la ley, debe ser la constante en el diseño de una estrategia tecnológica al servicio de la fuerza pública, para poder confrontar información sobre los individuos, pero sin vulnerar sus derechos humanos, y lograr ejercer un control actuarial sobre los individuos para disminuir su acción criminal.

El conflicto armado que persiste en la actualidad en Colombia, va a requerir estrategias inmediatas porque aún no se ha terminado, y en el caso de que se termine bien sea por acción militar o política, se debe continuar con la misma estrategia tecnológica de control actuarial, puesto que los individuos que no pertenecían a una estructura militar de las guerrillas, quedan a la deriva y bajo su propio control y autonomía, lo cual hace que la fuerza pública no eluda esa responsabilidad para garantizar la seguridad y la tranquilidad ciudadanas que es un mandato constitucional.

No se trata de distribuir la responsabilidad para que las áreas urbanas y suburbanas queden en cabeza de la Policía Nacional y las áreas rurales en las Fuerzas Militares, sino obtener el éxito en el diseño de una estrategia de control actuarial para que las acciones de la fuerza pública se integren con una mayor eficacia sobre las diferentes organizaciones al margen de la ley que delinquen y comparten las dos características de concentración de población (rural, urbana), y se pueda combatir todas las formas de delito con una mayor agilidad, y generar con ello la credibilidad y la confianza por parte de la población (Bergalli, 2001), legitimando el profesionalismo de una fuerza pública proactiva.

El Actuarialismo tiene como esencia, gestionar la afectación del ambiente para evitar que desde allí, los grupos delincuenciales al margen de la ley generen acciones criminales, para lo cual se requiere del control actuarial sobre los individuos como agentes gestores del mismo. Por consiguiente, se debe desarrollar estrategias y diseños, implementando tecnologías e informática para contrarrestar la acción criminal que deben concentrar información sobre el comportamiento de los potenciales criminales, para anticiparse a su neutralización. 
El desarrollo de las tecnologías (aplicaciones Apps y otras), se orienta a información sobre personas, su actividad económica, vehículos, residencia, coordenadas, ubicación específica, fotografía, material y transporte, combustibles, insumos químicos, etc. Esta información debe estar asegurada con directrices del mando y seguridad informática, para que su manejo se haga dentro de los protocolos de la legalidad. El manejo ágil de la información por parte de los diferentes comandantes, les va a facilitar la toma de decisiones oportunas y contribuir en el mejoramiento y desarrollo del profesionalismo y efectividad de la fuerza pública.

El control territorial en las áreas rurales se debe continuar por parte de las Fuerzas Militares, en el entendido de que la gran preocupación es que la periferia sea en primera instancia, la facilitadora de toda acción criminal producto de las acciones terroristas de las guerrillas contra la infraestructura energética y vial, centros económicos, bandas criminales, narcotraficantes con los cultivos, procesamiento y distribución de narcóticos, hacia los centros urbanos, convirtiéndolos en receptores de segunda instancia en la continuación y terminación de las infraestructuras criminales. La clandestinidad que puede garantizarles seguridad a los criminales para actuar inicialmente en el área rural, se puede neutralizar en las áreas urbanas con los controles actuariales tecnológicos y humanos que los mandos diseñen en el marco de la legalidad y oportunidad.

\section{REFERENCIAS BIBLIOGRÁFICAS}

Beck, U. (1998). La sociedad del riesgo. Hacia una nueva modernidad. Barcelona: Paidós Ibérica.

-------. (2002). La democracia y sus enemigos. Barcelona: Paidós Ibérica.

Bergalli, R. (2001). Globalización y control social: post-fordismo y control punitivo. En: El Sistema. Revista de Ciencias Sociales 160. Madrid.

Comando General de las Fuerzas Militares (1996). Manual de Seguridad y Defensa Nacional. Bogotá: Imprenta de las Fuerzas Militares.

De Giorgi, A. (2004). Tolerancia Cero. Estrategias y prácticas de la sociedad del control. Barcelona: Virus.

Feeley, M. y Simon, J. (1995). La nueva penología: notas acerca de las estrategias emergentes en el sistema penal y sus implicaciones, Delito y Sociedad. En: Revista de Ciencias Sociales. Año 4, 6. Santa Fe: Universidad Nacional del Litoral.

Leal, J. (2004). Delito y modernidad. México: Fontamara.

Leal, F. (2003). La doctrina de seguridad nacional: materialización de la guerra fría en América del Sur. En: Revista de Estudios Sociales 15, junio 2003., pp. 74-87.

Ministerio de Defensa Nacional (2006). Logros de Política Integral de seguridad y Defensa para la Prosperidad. Dirección de Estudios estratégicos. En: http://www.mindefensa.gov.co/irj/go/km/docs/Mindefensa/Documentos/descargas/estudios\%20sectoriales/info_estadistica/Logros_Sector_Defensa.pdf

Naim, M. (2014). Vladimir el débil. En: El Tiempo, 23 de marzo. En: http://www.eltiempo.com/opinion/columnistas/vladimir-el-debil/13711741 (12 junio 2014).

Novoa, J. (2012). Actuarialismo penitenciario en América Latina. En: Revista de Ciencias Sociales 7, pp. 65-80. En: http:// www.unilibrectg.edu.co/Descarga/PDF/SABER_CIENCIAYLIB_ENE_JULIO_2012.pdf (22 abril 2013). 
(2013). La Cuestión Integral del siglo XXI. Actuarial, Seguridad, Criminal, Cárcel. En: Revista Educación y Desarrollo Social. Vol. 7, 2., pp. 166-185.

O’Malley, P. (2004). Riesgo, Poder y Prevención del Delito. Traductores: M. Sozzo y A. Montero. En: Prevenir el delito más allá de la pena. Buenos Aires: Ad-Hoc.

Patiño, C. (2013). Guerras que cambiaron al mundo. Bogotá: Random House Mondadori.

Rivera, I. (2009). Cuestión carcelaria. Historia, epistemología, derecho y política penitenciaria. Buenos Aires: Del Puerto.

Rutherford, A. (2000). An Elephant on the Doorstep: Criminal Policy without Crime in New Labour's Britain. Oxford: Hart Publishing., pp. 81-112.

Torres, C., y Peruzzotti, E. (2008). El retorno del pueblo. Populismos y nuevas democracias en América Latina. Quito: FLACSO.

Waltz, K. (2007). El hombre, el Estado y la guerra. En: Revista Académica de Relaciones Internacionales 6. Abril., p. 20.

Weber, M. (1994). Economía y Sociedad. Esbozo de sociología comprensiva. México: Fondo de Cultura Económica. 
